\title{
Evaluation of Theoretical Velocities and Comparative Study of Ultrasonic Velocities in Binary Liquid Mixtures of Ethyl Lactate with Branched Alkanols at Different Temperatures
}

\author{
K. A. K. RAJKUMAR ${ }^{1}$, G. R. SATYANARAYANA ${ }^{2}$, G. LAKSHMANA RAO ${ }^{1}$, \\ SK. BEEBI ${ }^{1}$, P. B. SANDYA SRI ${ }^{3}$ and C. RAMBABU ${ }^{1 *}$ \\ ${ }^{1}$ Department of Chemistry, Acharya Nagarjuna University, Nagarjuna Nagar, Guntur, \\ Andhra Pradesh, India \\ ${ }^{2}$ Department of Chemistry, Sir. C.R. Reddy PG College, Eluru, Andhra Pradesh, India \\ ${ }^{3}$ Department of Physics, K.B.N. College, Vijayawada-, Andhra Pradesh, India \\ rbchintala@gmail.com
}

Received 15 October 2017 / Accepted 6 November 2017

\begin{abstract}
Theoretical velocities of binary liquid mixtures of ethyl lactate (EL) with 2-propanol (PNL), 2-butanol (BNL) and 2-methy,1-propanol (MPL), at different temperature (303.15, 308.15, 313.15 and $318.15 \mathrm{~K}$ ) have been evaluated by using theoretical models of Nomoto (NOM), Impedance (IMP), Van Dael and Vangeel (VDV), Junjie's (JUN) and Rao's specific velocity (RAO) relations. Ultrasonic velocities and densities of these mixtures have also been measured experimentally as a function of composition of EL and temperature. The deviation in experimental values and calculated values from different theories confirms the existence of molecular interactions between molecules of the constituent liquids. $\mathrm{U}_{\text {exp }}^{2} / \mathrm{U}^{2}$ imx has also been evaluated for non-ideality in the mixtures. Chi-square test for the goodness of the fit is applied to investigate the relative applicability of these theories to the present systems. The results are subjected to discussion in terms of intermolecular interactions between the component molecules in the chosen binary liquid mixtures.
\end{abstract}

Keywords: Theoretical velocities, Ultrasonic velocities, Chi-square test, Molecular interaction parameter

\section{Introduction}

In the recent years, ultrasonic studies play an important role in various organic liquid mixtures ${ }^{1-4}$ due to the fact that the optical methods cannot identify and assess all types of interactions in the liquid mixtures having weak interactions. The physicochemical properties like adiabatic compressibility, heat capacity, coefficient of expansion may be obtained from ultrasonic velocity, density and viscosity data. The molecular interactions in pure and binary liquid mixtures can be qualitatively analyzed using ultrasonic velocity measurements which 
are of considerable interest in the last several years, using various theories ${ }^{5-9}$, ultrasonic sound velocities in liquid mixtures have been calculated and compared with experimental values. The present work is a continuation of our research programme on a comparison of experimental ultrasonic velocity with the theoretical models of Nomoto, impedance relation, Van Dael ideal mixing relation, Rao's specific velocity and Junjie's relation for the binary mixtures of several systems at various temperatures by our researchers. The results are interpreted in terms of intermolecular interactions between the binary component liquid mixtures ${ }^{10-18}$. In this paper, we report the experimental and theoretical ultrasonic velocities of the binary liquid mixtures of ethyl lactate with 2-propanol, 2-butanol and 2-methyl,1-propanol at 303.15, 308.15, 313.15 and $318.15 \mathrm{~K}$ over the entire composition range, evaluated by using various theoretical models such as Nomoto (NOM), impedance (IMP), Van Dael and Vangeel (VDV), Junjie's (JUN) and Rao's specific velocity (RAO) relations. Further, a comparative study of theoretical results with experimental values using Chi-square test and the study of molecular interactions from the deviation $(\alpha)$ in the value of $\mathrm{U}^{2} \exp / \mathrm{U}^{2}$ imx (from unity) has also been made.

\section{Experimental}

Commercially available pure solvents were used in the present investigation EL (Merk > 99\%) and PNL, BNL, MPL of AR grade procured from S.D fine chemicals (India) were purified by the standard methods described by Weissberger ${ }^{19,20}$ and the purity of the chemicals was assessed by comparing their measured densities $(\rho)$ and ultrasonic velocities $(U)$ which are in good agreement with literature values. The mixtures were prepared gravimetrically using an electronic balance (Shimadzu AY120) with an uncertainty of $\pm 1 \times 10^{-7} \mathrm{Kg}$ and were stored in airtight glass bottles. The uncertainty in the mole fraction was estimated to be less than $\pm 1 \times 10^{-4}$. It is ensured that the components were adequately mixed before being transferred in to the apparatus. The required properties are measured within one day of the mixture preparation.

The densities, $\rho$, of pure liquids and their mixtures are determined for evaluating mole fractions using a $10^{-5} \mathrm{~m}^{3}$ Double - arm pycnometer and the values from triplicate replication at each temperature are reproducible within $2 \times 10^{-1} \mathrm{~kg} \mathrm{~m}^{3}$ and the uncertainty in the measurement of density is found to be 2 parts in $10^{4}$ parts. The reproducibility in mole fractions is within \pm 0.0002 . Temperature control for the measurement of viscosity and density is achieved by using a microprocessor assisted circulating water bath, (supplied by Mac, New Delhi) regulated to $\pm 0.01 \mathrm{~K}$, using a proportional temperature controller. Adequate precautions are taken to minimize evaporation losses during the actual measurements. The ultrasonic velocity of sound (U) is measured using an ultrasonic interferometer (Mittal Enterprises, New Delhi model F05) operating at $2 \mathrm{MHz}$. The measured speeds of sound have a precision of $0.8 \mathrm{~m} . \mathrm{s}^{-1}$ and an uncertainty less than $\pm 0.1 \mathrm{~m} . \mathrm{s}^{-1}$. The temperature stability is maintained within $\pm 0.01 \mathrm{~K}$.by circulating water bath around the measuring cell through a pump.

\section{Theoretical considerations}

\section{Nomoto theory}

Nomoto's empirical formula is based on the assumption of the linear dependence of the molecular sound velocity on concentration and the additivity of the molar volume in the liquid mixture. The sound velocity $\mathrm{U}$ is given by

$$
\mathrm{U}=\left[\frac{\sum_{i=1}^{n} x_{i} R_{i}}{\sum_{i=1}^{n} x_{i} V_{i}}\right]^{3}
$$


Where the molar sound velocity $\mathrm{R}=\mathrm{x}_{1} \mathrm{R}_{1}+\mathrm{x}_{2} \mathrm{R}_{2}$. Hence, ultrasonic velocity $(\mathrm{U})$ is given by

$$
\mathrm{U}=\left[\frac{x_{1} R_{1}+x_{2} R_{2}}{x_{1} V_{1}+x_{2} V_{2}}\right]^{3}
$$

In the above equation $\mathrm{R}_{\mathrm{i}}=\left(\mathrm{M}_{\mathrm{i}} / \rho_{\mathrm{i}}\right) \mathrm{U}_{\mathrm{i}}^{1 / 3}=\mathrm{V}_{\mathrm{i}}\left(\mathrm{U}_{\mathrm{i}}\right)^{1 / 3}$

\section{Impedance relation}

The specific acoustic impedance of the pure liquids was used for evaluating the ultrasonic velocity in the liquid mixtures by the following relation:

$$
\mathrm{U}=\sum \mathrm{x}_{\mathrm{i}} \mathrm{Z}_{\mathrm{i}} / \sum \mathrm{x}_{\mathrm{ii}}
$$

Where $Z_{\mathrm{i}}$ is acoustic impedance and $\rho_{\mathrm{i}}$ is the density of the mixture.

\section{Van Dael and Vangeel relation}

Van Dael and Vangeel obtained the formula for ultrasonic velocity in the liquid mixtures adopting the adiabatic compressibilities of the pure liquids based on ideal mixing of the liquids. Van Dael and Vangeel assumed that the adiabatic compressibility $\left(\beta_{\mathrm{ad}}\right)$ of the mixture is given by

$$
\beta_{\mathrm{ad}}=\phi_{\mathrm{A}}\left(\beta_{\mathrm{ad}}\right)_{\mathrm{A}}+\phi_{\mathrm{B}}\left(\beta_{\mathrm{ad}}\right)_{\mathrm{B}}
$$

and suggested the following relation for sound velocity in homogeneous liquid mixtures.

$$
\beta_{a d}^{i m}=\phi_{\mathrm{A}} \frac{\gamma_{\mathrm{A}}}{\gamma^{i m}}\left(\beta_{a d}\right)_{A}+\phi_{\mathrm{B}} \frac{\gamma_{\mathrm{B}}}{\gamma^{i m}}\left(\beta_{a d}\right)_{B}
$$

Where $\phi$ and $\gamma$ refer the volume function and principal specific ratio.

It holds true if the mixture is an ideal one and also $\gamma_{A}=\gamma_{B}=\gamma_{i m}$. It can be transformed into a linear combination of the mole fractions if the additional assumption $\mathrm{v}_{\mathrm{A}}=\mathrm{v}_{\mathrm{B}}$ is made

$$
\beta_{\mathrm{ad}}^{\mathrm{im}}=\mathrm{x}_{\mathrm{A}}\left(\beta_{\mathrm{ad}}\right)_{\mathrm{A}}+\mathrm{x}_{\mathrm{B}}\left(\beta_{\mathrm{ad}}\right)_{\mathrm{B}}
$$

The sound velocities appropriate to the above equations are given by

$$
\begin{aligned}
& \frac{x_{A} v_{A}+x_{B} v_{B}}{x_{A} M_{A}+x_{B} M_{B}} \frac{1}{\left(U^{i m}\right)^{2}}=\varphi_{A} \frac{v_{A}}{M_{A} U_{A}^{2}}+\varphi_{B} \frac{v_{B}}{M_{B} U_{B}^{2}} \quad \text { and } \\
& \frac{1}{x_{A} M_{A}+x_{B} M_{B}} \frac{1}{\left(U^{i m}\right)^{2}}=\frac{x_{A}}{M_{A} U_{A}^{2}}+\frac{x_{B}}{M_{B} U_{B}^{2}}
\end{aligned}
$$

\section{Junjie's relation}

This relation derived by Junjie's for the ultrasonic velocity of the mixture in terms of the mole fraction, molecular weight and density of the mixture.

$$
U=\frac{\sum_{i=1}^{n} x_{i} V_{i}}{\left(\sum_{i=1}^{n} x_{i} M_{i}\right)^{1 / 2}\left(\sum_{i=1}^{n} x_{i} V_{i} / \rho_{i} u_{i}^{2}\right)^{1 / 2}}
$$

Where the symbols have their usual meanings. 


\section{Rao's relation}

Using the ratio of the temperature coefficient of velocity and expansion coefficient, Rao derived a formula for ultrasonic velocity (U)

$$
\mathrm{U}=\left(\frac{R}{V}\right)^{3}
$$

Where V is the molar volume and $\mathrm{R}$ is called Rao's constant or molar sound velocity, which is constant for a liquid at a temperature.

\section{Chi-square test for goodness of fit}

According to Karl Pearson, Chi-square value is evaluated for the binary liquid mixtures under study using the formula

$$
x^{2}=\sum_{i=1}^{n}\left(\left(U_{(o b s)}-U_{(c a l)^{2}} / U_{(C a l)}\right.\right.
$$

Where $\mathrm{n}$ is the number of data used and ' $\mathrm{U}_{(\mathrm{obs})}=$ experimental values of ultrasonic velocities, $\mathrm{U}_{(\mathrm{cal})}=$ computed values of ultrasonic velocities

\section{Relative percentage of error $(\sigma)$}

The Average percentage error is calculated by using the relation

$$
\sigma=1 / \mathrm{n} \sum\left(\left(\mathrm{U}_{(\mathrm{obs})}-\mathrm{U}_{(\mathrm{cal})}\right) / \mathrm{U}_{(\mathrm{obs})}\right) \mathrm{X} 100 \%
$$

Where $\mathrm{n}$ is the number of data used.

\section{Molecular associations}

The degree of intermolecular interaction or molecular association is given by

$$
\alpha=\left[\mathrm{U}^{2}{ }_{\text {exp }} / \mathrm{U}^{2}{ }_{\text {imx }}\right]-1
$$

Where $U_{\text {exp }}$ and $U_{\text {imx }}$ are the experimental and theoretical velocities.

\section{Results and Discussion}

The experimental ultrasonic velocities and the theoretical values evaluated by Nomoto's Relation (NOM), Impedance Relation (IMP), Van Deal and Vangeel Ideal Mixing Relation (VDV), Junjie's relation (JUN), Rao's specific velocity method (RAO) for all the three binaries ethyl lactate +2 -propanol, ethyl lactate +2 -butanol and ethyl lactate +2 -mthyl,1propanol along with the percentage of deviations are presented in Tables 1-3 at all the four temperatures $303.15,308.15,313.15$ and $318.15 \mathrm{~K}$ and atmospheric pressure. The validity of different theoretical formulae is checked by the chi-square test for all the mixtures at all the temperatures and the values are given in Table 4.

Table 1. Experimental and theoretical values of velocities with their \% deviations for the system $(\mathrm{EL}+\mathrm{PNL})$

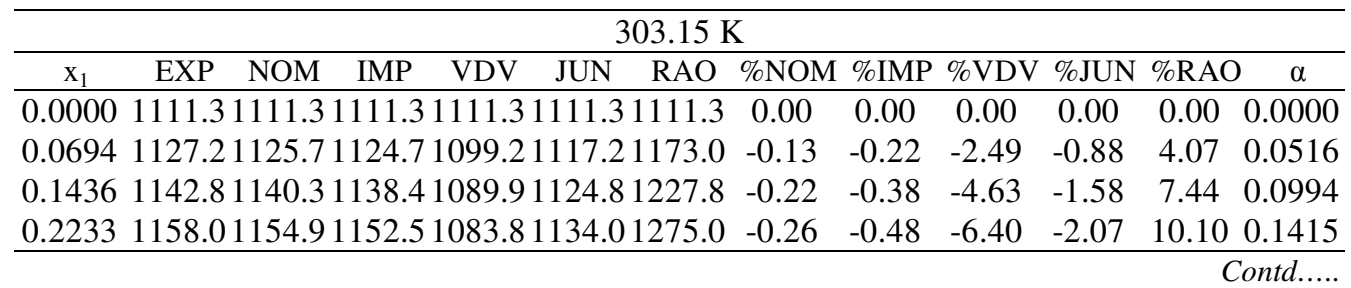




\begin{tabular}{|c|c|c|c|c|c|}
\hline 901 & .20 & -0.52 & -7.79 & -2.38 & .93 \\
\hline & -0.26 & -0.52 & -8.72 & & \\
\hline .7 & -0.24 & -0.49 & .11 & 14 & \\
\hline 1217.21214 .91212 .21110 .01 & -0.19 & 41 & 81 & .17 & 2026 \\
\hline 0.72851231 .71230 .21228 .11138 .61211 .01344 .3 & -0.12 & -0.29 & -7.56 & 1.68 & 9.14 \\
\hline 85791246.71245 .612 & -0.09 & -0.18 & -4.94 & -0.99 & 5.26 \\
\hline 1.000012 & 0 & & 0.00 & 0.00 & 0.00 \\
\hline \multicolumn{6}{|l|}{$308.15 \mathrm{~K}$} \\
\hline $\begin{array}{lll}\text { EXP } & \text { NOM } & \text { IMP } \\
\end{array}$ & $\mathrm{NOM}$ & L & $6 \mathrm{VDV}$ & $\% \mathrm{JUN}$ & $\%$ RAO \\
\hline 06.41106 .41106 .411 & 0.00 & 0.00 & 0.00 & 0.00 & 0.00 \\
\hline 120.21119 .51118 .61094 .011 & 0.06 & 14 & -2.34 & -0.77 & 4.20 \\
\hline 0 & -0.12 & 28 & -4.39 & -1.40 & 7.680 \\
\hline 0.2 & -0.14 & & -6.09 & -1.85 & 10.420 \\
\hline 0.3 & -0.10 & & -7.37 & -2.09 & 12.360 \\
\hline 0.4 & -0.09 & -0.33 & -8.27 & -2.20 & 13.340 \\
\hline 1184.01084 .911 & -0.06 & -0.30 & -8.65 & -2.13 & 13.260 \\
\hline & -0.01 & & -8.33 & -1.86 & 12.030 \\
\hline & -0.03 & & -7.19 & -1.48 & 9.43 \\
\hline & 0.01 & & -4.66 & -0.84 & 5.48 \\
\hline 6 & .00 & & 0.00 & 0.00 & $0.00 \quad 0.0000$ \\
\hline \multicolumn{6}{|c|}{$313.15 \mathrm{~K}$} \\
\hline $\begin{array}{lll}\text { EXP } & \text { NOM } & \text { IMP } \\
\end{array}$ & NOM & \%IMP & $\% \mathrm{VDV}$ & $\% \mathrm{JUN}$ & $\%$ RAO \\
\hline 00001097.21097 .21097 .210 & $\overline{00}$ & & 0.00 & 0.00 & $\overline{000}$ \\
\hline 641 & 01 & & .22 & -0.67 & 459 \\
\hline .5 & .02 & 12 & -4.15 & -1.20 & 7.950 \\
\hline 0.223311 & 0.02 & -0.17 & -5.79 & -1.61 & 10.730 .1268 \\
\hline 0.30901 & 08 & 5 & 7.03 & 1.82 & 12 \\
\hline 1316.7 & .11 & 13 & -7.89 & -1.91 & 13.730 \\
\hline 0.50151170 .21171 .71169 & 0.13 & -( & -8.26 & -1.85 & 13 \\
\hline & 18 & 02 & -7.95 & -1.59 & 12.40 \\
\hline & & & -6 & 25 & $9.75 \quad 0.1522$ \\
\hline 912 & & & -4.44 & -0.71 & $5.64 \quad 0.0952$ \\
\hline .21223 .2 & 0.00 & & 0.00 & 0.00 & $0.00 \quad 0.0000$ \\
\hline \multicolumn{6}{|l|}{$318.15 \mathrm{~K}$} \\
\hline $\begin{array}{llll}\text { EXP } & \text { NOM } & \text { IMP } & \text { VDV } \\
\end{array}$ & $\mathrm{NOM}$ & $\underline{\text { MP }}$ & $\% \mathrm{VDV}$ & bJUN & $\% \mathrm{RAO}$ \\
\hline 0.00001090 .11090 .11090 .11090 .11090 .11090 .1 & 0.00 & & 0.00 & 0.00 & $0.00 \quad 0.0000$ \\
\hline 0.069411 & 0.09 & 1 & -2.08 & -0.56 & $4.54 \quad 0.0428$ \\
\hline 31110.81067 .31099 .31202 .0 & 0.18 & & -3.88 & -0.99 & $8.26 \quad 0.0823$ \\
\hline 311 & 0 . & & -5 & -1.28 & 90. \\
\hline 0.30901 & 0.31 & 1 & -6.59 & -1.49 & 13.210 .1461 \\
\hline 0.401411 & 0 . & & -7.40 & -1.54 & 14.260 .1661 \\
\hline 0.501511 & & & -7.75 & -1.48 & 0.1750 \\
\hline .61314 .1 & 0.41 & 0.22 & -7.46 & -1.26 & 12.870 .1678 \\
\hline 0.72851176 .3118 & 0.37 & & -6.38 & -0.93 & 10.180 .1409 \\
\hline 31183.41259 .9 & 0.23 & & -4.14 & -0.52 & $\begin{array}{ll}5.91 & 0.0883\end{array}$ \\
\hline 1.00001204 .11204 .11204 .11204 .11204 .11204 .1 & 0.00 & 0.00 & 0.00 & 0.00 & $0.00 \quad 0.0000$ \\
\hline
\end{tabular}


Table 2. Experimental and theoretical values of velocities with their $\%$ deviations for the system (EL+BNL)

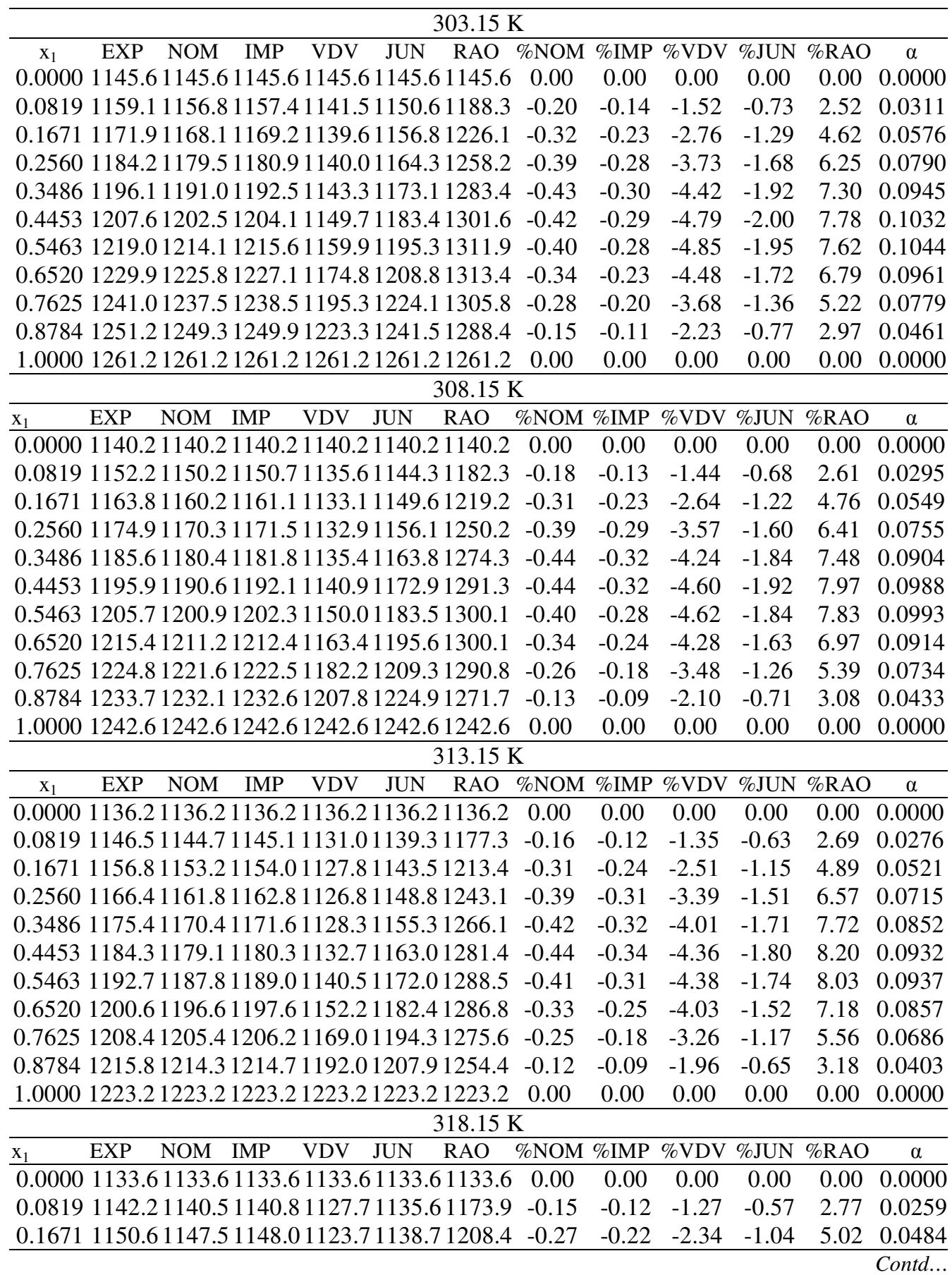




\begin{tabular}{|c|c|c|c|c|c|c|}
\hline & 36 & -0.30 & -3.17 & -1.37 & 6.72 & 6 \\
\hline 1257.9 & 0.43 & -0.36 & -3.79 & -1.60 & 7.83 & 0803 \\
\hline 71.4 & .44 & -0.36 & -4.10 & -1 & 33 & 0873 \\
\hline 76.8 & -0.39 & -0.33 & -4.10 & -1.58 & 8.19 & 874 \\
\hline .411 & -0.33 & -0.27 & -3.77 & -1.38 & 7.32 & 0.0798 \\
\hline 01260.2 & -0.20 & -0.16 & -3.01 & -1.02 & 5.70 & 0.0630 \\
\hline 7.4 & -0.08 & -0.06 & -1.78 & -0.55 & 3.30 & 0.0366 \\
\hline 1.00001204 .11204 .11204 .11204 .11204 .11204 .1 & 0.00 & 0.00 & 0.00 & 0.00 & 0.00 & 0.0000 \\
\hline
\end{tabular}

Table 3. Experimental and theoretical values of velocities with their \% deviations for the system (EL+MPL)

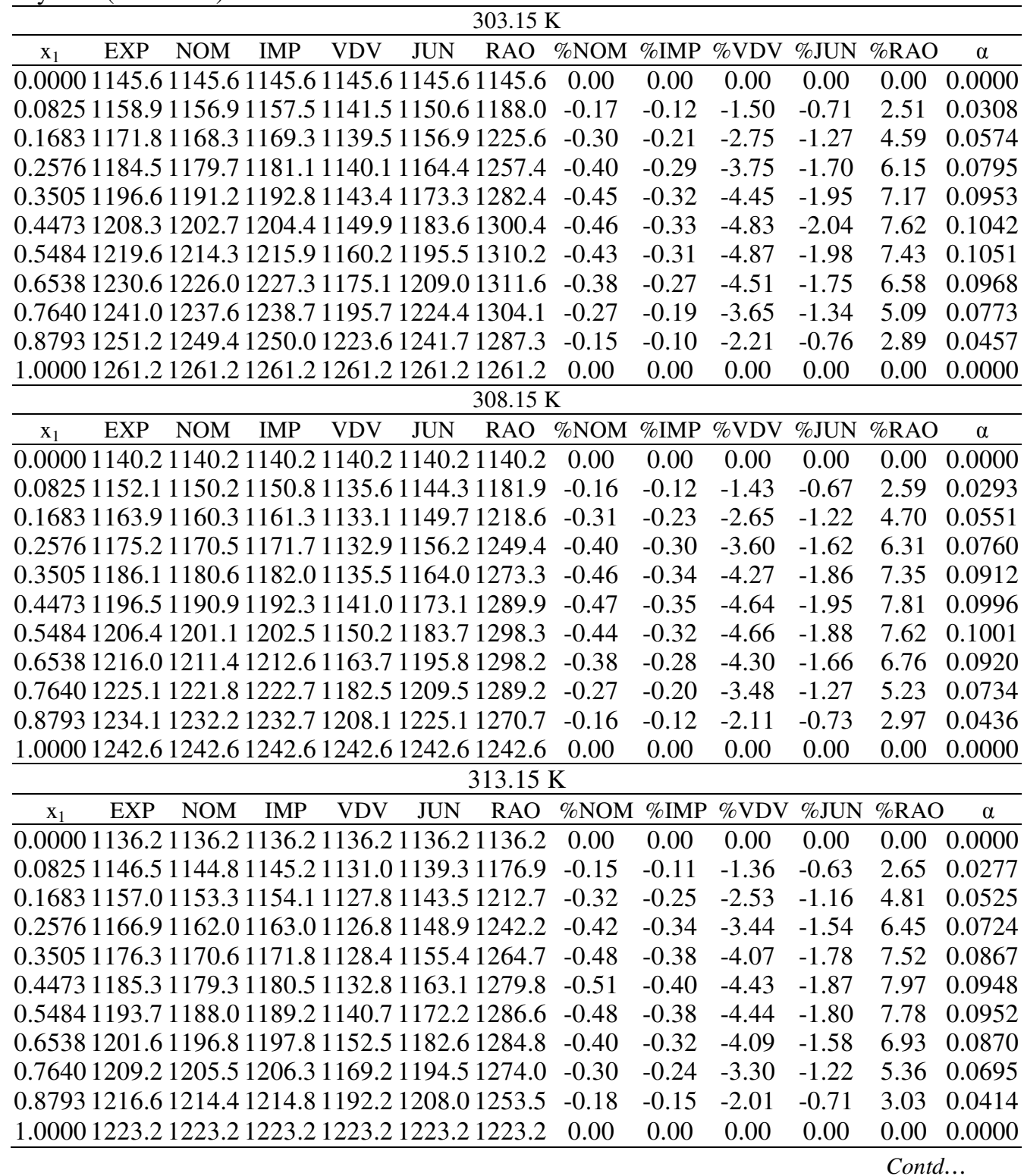




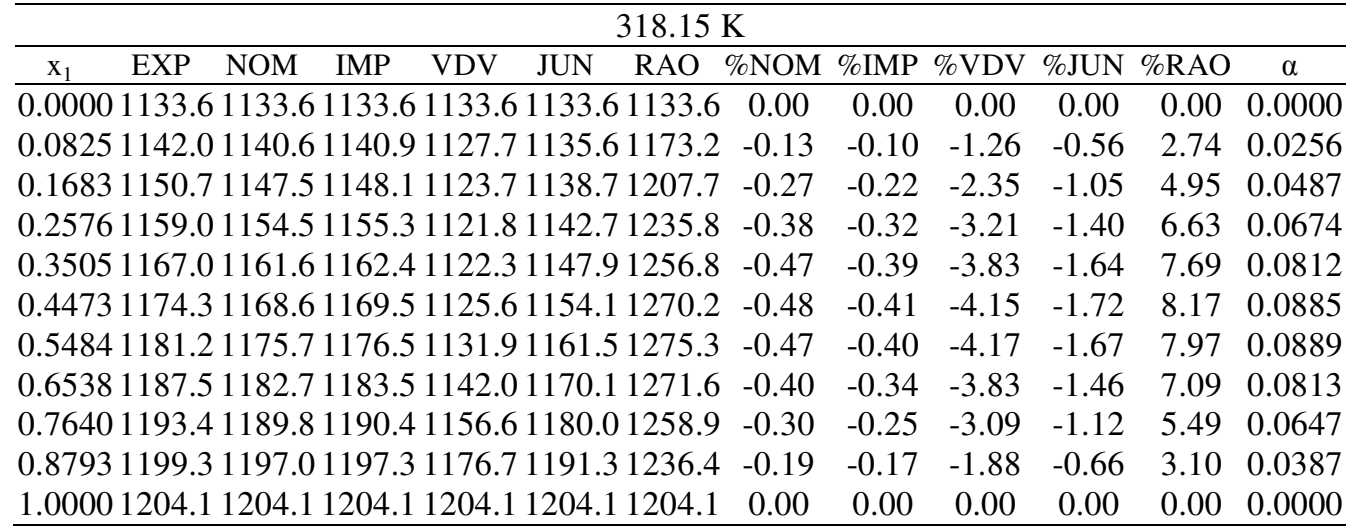

Table 4. Values of $\chi^{2}$ and $\sigma$ for all values of the 3 binary mixtures at 4 different temperatures system-I (EL+PNL)

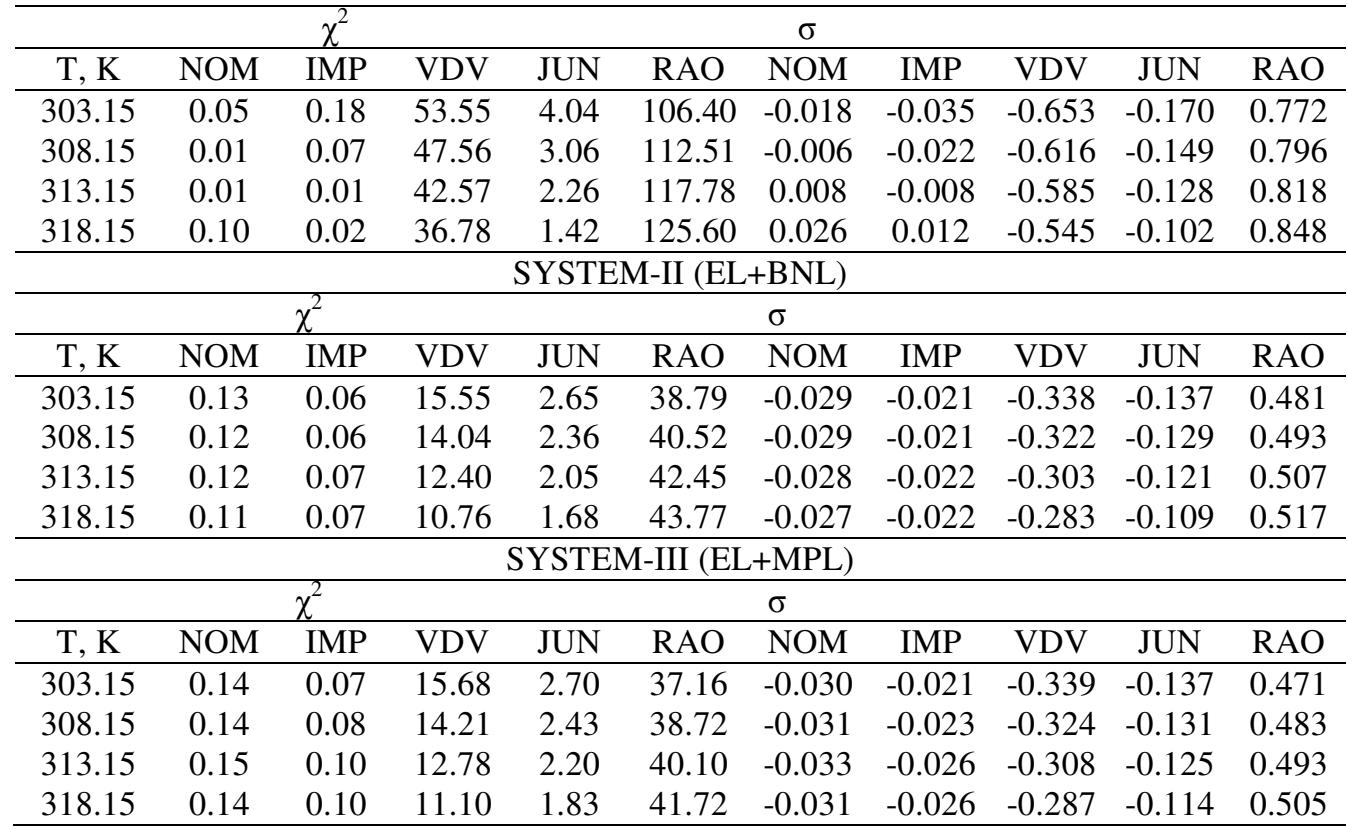

Even though, there are variations between the evaluated and experimental values for all the theoretical models, the deviations in velocities for Impedance relation (IMP) and Nomoto models (NOM) are found to be less than that of other approaches at all temperatures. The occurrence of positive deviations in all the models confirms the existence of molecular interactions due to strong hydrogen bonding interactions between the components ${ }^{21}$. There are larger variations in some intermediate concentration range in all the models suggesting the existence of strong tendency of association between component molecules as a result of hydrogen bonding. Nomoto's theory proposes that the volume does not change upon mixing. Therefore, no interaction between the components of liquid mixtures has been taken into account. Similarly, the assumption for the formation of ideal mixing relation is that, the ratios of specific heats of ideal mixtures and the volumes are also equal. Again no molecular 
interactions are taken into account. But upon mixing, interactions between the molecules occur because of the presence of various types of forces such as dispersion forces, charge transfer, hydrogen bonding dipole-dipole and dipole-induced dipole interactions resulting in the deviations.. Therefore, it is attributed that the observed deviation of theoretical values of velocity from the experimental values arise due to the molecular interactions between the unlike molecules in the liquid mixtures. From the tables, it is observed that maximum deviation at 0.5 mole fraction of all the 3 systems at all the temperatures. The ratio $\mathrm{U}^{2}{ }_{\text {exp }} / \mathrm{U}^{2}{ }_{\mathrm{imx}}$ is an important tool to measure the non ideality in the mixtures especially in such cases where the properties other than sound velocity are not known. The percentage deviations in velocity are found to have both negative and positive magnitudes indicating the non ideal behaviour of liquid mixtures. The evaluated interaction parameters are positive for all the systems, indicating stronger interactions between the mixing molecules, which increase from PNL to MPL. This suggests somewhat stronger interaction of EL with PNL in comparison to other components. The negative values indicate the dominance of dispersion forces arising from the breakage of hydrogen bonds in the associates. But a positive value of $\alpha$ in all the system clearly indicates the existence of strong tendency for the formation of association in mixture through dipole-dipole interactions higher values of percentage deviation indicates maximum departure of the particular theory from experiment at that particular concentration and magnitude of the chi-square value finally determines the overall validity of the theory. The chi square values along with average percentage error are given in Table 4 .

\section{Conclusion}

From the values of experimental and evaluated velocity values, it may be concluded that, the Impedance Relation followed by Nomoto relation of Ultrasonic velocity have provided results with smaller deviations. Thus, the linearity of molar sound velocity and additivity of molar volumes, as suggested by Impedance Relation and Nomoto relation in deriving the empirical relations have not been truly observed in the aforementioned binary liquid mixtures. However, the positive deviations of the experimental velocities from the theoretical values using all the theoretical models indicate the existence of chemical forces such as hydrogen bonding and dipole-dipole interactions between the component molecules.

\section{References}

1. Ali A, Yasmin A and Nain A K, Indian J Pure Appl Phys., 2002, 40, 315-322.

2. Ali A A, Nain A K and Soghra Hyder, J Pure Appl Ultrason., 2001, 23, 73.

3. Vasantharani P, Muthu Shailaja S, Kannappan A K and Ezhil Pavai R, J Appl Sci., 2008, 8(12), 2329-2332; DOI:10.3923/jas.2008.2329.2332

4. Satyanarayana G R, Kumar D B K, Sujatha K, Lakshmanarao G and Rambabu C, $J$ Molec Liq., 2016, 216, 526-537; DOI:10.1016/j.molliq.2016.01.054

5. Nomoto O, J Phys Soc Japan, 1958, 13(12), 1528-1532; DOI: 10.1143/JPSJ.13.1528

6. Baluja S and Parsania P H, Asian J Chem., 1955, 7(2), 417-423.

7. Van Dael W and Vangeel E, Pro Int Conf on Calorim Therm., Warsaw, 1955, 555.

8. Junjie Z, J China Univ Sci Technol., 1984, 14, 298.

9. Rama Rao M, J Chem Phys., 1941, 9(9), 682; DOI:10.1063/1.1750976

10. Satyanarayana G R, Krishna K B M, Sujatha K and Rambabu C, Der Pharma Chemica, 2014, 6(5),158-165.

11. Lakshmi B J, Sankar M G, Begum Z, Ramachandran D and Rambabu C, Elixir Ultrasonics, 2013, 65, 19808-19814. 
12. Lakshmi B J, Satyanarayana G R, Sankar M G, Ramachandran D and Rambabu C, Chem Sci Trans., 2015, 4(1), 940-950; DOI:10.7598/cst2015.940

13. Satyanarayana G R, Sandya Sri P B, Sujatha K and Rambabu C, Elixir Appl Chem., 2014, 77, 29018-29023.

14. Rayapa Reddy K, Bala Karuna Kumar D, Rambabu C and Srinivasa Rao G, E-J Chem., 2012, 9(2), 553-562; DOI:10.1155/2012/571521

15. Bala Karuna Kumar D, Rayapa Reddy K, Srinivasa Rao G, Rama Rao G V and Rambabu C, Asian J Chem., 2012, 24(5), 2239-2244.

16. Sandhya Sri P B, Zareena Begum and Rambabu C, J Thermodyn Catal 2013 4, 120; DOI10.4172/2157-7544.1000120

17. Rajkuma K A K, Satyanarayana G R, Lakshmanrao G, Beebi S K, Bala D and Rambabu C, Chem Sci Trans., 2017, 6(1), 87-96; DOI:10.7598/cst2017.1325

18. Lakshmana Rao G, Sandhya Sri P B, Satyanarayana G R and Rambabu C, Der Pharma Chemica, 2015, 7(10), 398-408.

19. Weissberger, Proskaner E S, Riddick and Toops E E, Organic Solvents, 2, $2^{\text {nd }}$ Edn., Weissberger A, Wiley Interscience, New York, 1955.

20. Bunger W B, Reddick J A and Sankano T K, Organic Solvents, 3, 4 Edn., Weissberger A, Wiley Interscience, New York, 1986.

21. Satyanarayana G R, Sujatha K, Zareena Begum and Rambabu C, Phys Chem An Indian J., 2014, 9(8), 283-291. 